\title{
MODELO DE INTERIORIZACIÓN Y EXTERIORIZACIÓN PARA EXPLICAR EL INICIO DE LA PSICOPATOLOGÍA DE LOS TRASTORNOS ALIMENTARIOS EN LA ADOLESCENCIA
}

\author{
Alba Moreno Encinas', Jessica Moraleda Merino1, Montserrat Graell- \\ Berna², José Ramón Villa-Asensi², Tamara Álvarez', Tatiana Lacruz- \\ Gascón ${ }^{1}$ y Ana Rosa Sepúlveda García' \\ ${ }^{1}$ Universidad Autónoma de Madrid; ${ }^{2}$ Hospital Infantil Universitario Niño \\ Jesús, Madrid (España)
}

\begin{abstract}
Resumen
La etiología de los trastornos del comportamiento alimentario (TCA) es compleja y múltiples modelos han intentado conceptualizarla. El objetivo de este estudio fue determinar si los comportamientos interiorizados y exteriorizados son factores de riesgo generales de psicopatología o específicos de los TCA y qué componentes sintomatológicos del TCA están relacionados con estos comportamientos. Para ello, se utilizó un diseño transversal caso-control para comparar los TCA de inicio $(n=50)$ con tres grupos de control: un grupo con patología psiquiátrica (trastornos depresivos [TD], $n=40$ ), otro con patología psicosomática (asma, $n=40$ ) y un grupo control sin patología $(n=50)$. La muestra está compuesta por 180 mujeres adolescentes de 12-18 años y sus familias emparejadas por edad y estatus socioeconómico. Los resultados obtenidos indican que la interiorización es un factor de riesgo general para la psicopatología de TCA y TD, y que en los TCA está asociada específicamente con la obsesión por la delgadez, la ineficacia, la conciencia interoceptiva, la depresión, la ansiedadrasgo y la sintomatología obsesivo-compulsiva. Así, se entiende la elevada comorbilidad de los TCA con otros comportamientos interiorizados.

PALABRAS CLAVE: factores de riesgo, trastornos del comportamiento alimentario, comportamientos interiorizados, comportamientos exteriorizados, adolescentes.
\end{abstract}

\footnotetext{
El proyecto "ANOBAS" se inició con el contrato Ramón y Cajal (Dra. Sepúlveda, RYC-2009-05092) y ha recibido también financiación por el Ministerio de Ciencia e Innovación, Plan Nacional I +D+ i 2008/11 (PSI2011-23127). Nos gustaría agradecer a las familias, psiquiatras y otros profesionales de salud mental en el proceso de reclutamiento, principalmente a la Dra. E. Mollejo del Hospital del Sureste, Servicio de Salud Mental. También ha sido muy importante el apoyo de los directores y profesores de los siguientes centros de Secundarias: I.E.S. La Estrella, I.E.S. Las Musas, I.E.S. Alameda de Osuna. Nos gustaría agradecer la labor excelente de las psicólogas que han participado en las distintas etapas con las entrevistas de evaluación de las participantes, principalmente D. Anastasiadou, L. González y C. Bustos.

Correspondencia: Ana R. Sepúlveda, Facultad de Psicología, Dpto. Psicología Biológica y de la Salud, Universidad Autónoma de Madrid, Campus de Cantoblanco, 28049 Madrid (España). E-mail: anarosa.sepulveda@uam.es
} 


\begin{abstract}
The etiology of eating disorders (ED) is complex, and multiple models have attempted to conceptualize it. The objective of this study was to determine whether internalizing and externalizing behaviors are general proximal risk factors for psychopathology or specific for ED, and which symptomatic components of ED are related to these behaviors. Hence, a cross-case-control design was used to compare eating disorders at onset $(n=50)$ with three control groups: a group with psychiatric pathologies (depressive disorders [DD], $n=40$ ), a group with psychosomatic pathology (asthma, $n=40$ ), and a control group without pathologies $(n=50)$. The entire sample is made up of 180 adolescent women aged 12-18 years and their families matched by age and socioeconomic status. The results obtained indicate that internalization is a general risk factor for the psychopathology of ED and mood disorders, and that in ED it is specifically associated with a drive for thinness, ineffectiveness, interoceptive awareness, depression, trait-anxiety and obsessive-compulsive symptomatology. Thus, the high comorbidity of ED is associated mainly with other internalizing behaviors.

KEY WORDS: risk factors, eating disorders, internalizing behaviors, externalizing behaviors, teenagers.
\end{abstract}

\title{
Introducción
}

La psicopatología no tiene una causa única, sino que es el resultado de la influencia de múltiples factores psicológicos, biológicos y sociales. Por ello, conocer su etiología es especialmente complicado, ya que implica entender los procesos causales y los mecanismos de riesgo y protección que actúan a lo largo de la vida. Estos factores pueden ser específicos de un determinado trastorno, compartidos para varios trastornos, o incluso comunes a la mayor parte de los trastornos. La comorbilidad, es decir, el que en una misma persona se presenten dos o más trastornos, se puede explicar por dos razones. La primera es que haber sido expuesto a distintos factores de riesgo puede conducir al mismo resultado. Y la segunda, que un mismo factor de riesgo o circunstancia puede conducir a distintos efectos. Todo esto implica que en el curso del desarrollo cualquier factor puede provocar un efecto distinto en función de la organización del sistema en el que opera en cada momento (Toro y Ezpeleta, 2014).

Los factores de riesgo se definen como una variable que aumenta la probabilidad de que aparezca un trastorno psicológico posteriormente. Sin embargo, no implica causalidad, sino que se trata de una relación estadística de probabilidad entre dos variables, cuya importancia clínica se indica por la magnitud de la asociación. Los factores de riesgo proximales son aquellos que ocurren durante el primer año en que aparece el trastorno y precipitan su aparición. El número de factores de riesgo, el momento en que ocurren, la duración y el contexto son relevantes para entender el proceso causal, de modo que la importancia de un factor de riesgo debe ser entendida en relación con el estadio de desarrollo de la persona, el contexto en que ocurre y con qué otros factores interaccionan (Toro y Ezpeleta, 2014). Respecto a la etiología de los trastornos del comportamiento alimentario (TCA) se considera compleja y múltiples modelos han intentado conceptualizarla. A pesar de que se ha avanzado prolíficamente en su 
estudio no existe en la actualidad un acuerdo claro en cuáles son los factores de riesgo específicos de esta psicopatología (Jacobi, Hayward, Zwaan, Kraemer y Agras, 2004).

En concreto, los TCA se caracterizan por la preocupación por la imagen corporal, el peso, la talla y la forma, así como la alteración de los hábitos alimentarios o el control de peso. Esta alteración o las características asociadas del TCA dan como resultado un deterioro clínicamente significativo de la salud física o el funcionamiento psicosocial (American Psychological Association [APA], 2013). La incidencia global de los TCA ha aumentado en el grupo de alto riesgo (15-19 años), ya que cerca del $40 \%$ de los casos nuevos aparecen en esta edad (Smink, Van Hoeken y Hoek, 2012), siendo la anorexia nerviosa (AN) el subtipo de TCA más prevalente entre los adolescentes (Smink et al., 2012). El deterioro de las funciones y la comorbilidad con otros trastornos mentales son comunes en TCA. Se valora que el $50 \%$ de las personas con un TCA también cumplen los criterios para ansiedad y depresión (McDermott, Forbes, Harris, McCormack y Gibbon, 2006).

Ante este problema de la comorbilidad los modelos categoriales no han sabido responder de forma adecuada, y son los modelos dimensionales los que pretenden dar una respuesta a la elevada comorbilidad que existe entre determinados trastornos. En el DSM-5 se señala que "los abordajes diagnósticos dimensionales probablemente complementarán o sustituirán a los actuales planteamientos categóricos en los próximos años" (APA, 2013). Uno de los modelos dimensionales más extendidos actualmente es el modelo de interiorización y exteriorización (Achenbach y Rescorla, 2001), que podría ayudar a entender la alta comorbilidad de los TCA con otras psicopatologías en la adolescencia. Este modelo trata de dar una explicación etiológica a qué factores subyacentes dan lugar a la comorbilidad de los numerosos trastornos mentales y se refiere a dos grandes dimensiones de la psicopatología: la interiorización (INT), un factor general hacia el estado de ánimo cargado de afecto negativo y los problemas de ansiedad, y la exteriorización (EXT), un factor general hacia los conductas de desinhibición tales como el consumo de sustancias y la conducta antisocial, que forman patrones de comportamiento desadaptado (Cosgrove et al., 2011; Eaton et al., 2012). La manera más conocida de evaluar la INT y EXT es el "Sistema Achenbach de evaluación con base empírica" (Achenbach S"ystem of Empirically Based Assessment, ASEBA; Achenbach y Rescorla, 2001) que evalúa la psicopatología infantil y adolescente mediante la observación de los padres. La interiorización es la tendencia de las personas a expresar la angustia "hacia el interior/sí mismo", pues se trata de problemas relacionados con los estados internos. Hay trastornos categoriales que se englobarían dentro de la dimensión INT como los trastornos del estado de ánimo, los trastornos de ansiedad y los trastornos de quejas somáticas. Por el contrario, la exteriorización se trata de la tendencia de las personas a expresar la angustia "hacia el exterior/los demás" y se relaciona sobre todo con problemas en la conducta, que puede ser agresiva, hiperactiva, de delincuencia, entre otras. Entre los trastornos categoriales que se englobarían dentro de la dimensión EXT se incluyen el trastorno por déficit de atención e hiperactividad (TDAH), el trastorno negativista desafiante, 
el trastorno de conducta, el trastorno de personalidad antisocial y los trastornos por consumo de sustancias (Cosgrove et al., 2011; Eaton et al., 2012).

Existen pocos estudios que hayan estudiado estas dimensiones en TCA. De manera general, se han asociado los TCA con la dimensión INT (p. ej., Adambegan et al., 2012; Forbush et al., 2017; Forbush et al., 2010; Forbush y Watson, 2013; Hodge, Meilleur, Taddeo y Frappier, 2019; McDermott et al., 2006; Mitchell, Wolf, Reardon y Miller, 2014; Muratori, Viglione, Maestro y Picchi, 2004). En el estudio de Adambegan et al. (2012) las adolescentes que desarrollaron un AN en comparación con sus hermanas sanas tenían más problemas de comportamiento INT, en particular, problemas de aislamiento social (timidez y menos comunicativas) y síntomas depresivos con quejas de nerviosismo, soledad, inferioridad y culpa; mientras que las pacientes que desarrollaron bulimia nerviosa (BN), presentaban además comportamientos EXT premórbidos, como más disputas, inestabilidad emocional, celos y búsqueda de atención. En el estudio de McDermott et al. (2006) se encontraron niveles más bajos de psicopatología total y EXT, pero niveles similares de INT, en el grupo con TCA versus otra patología psiquiátrica. Además, se encontraron diferencias en la psicopatología general entre los subtipos de TCA, con tasas menores en el grupo de AN y con una psicopatología más grave y diversa (INT y EXT) en el grupo de TCA no especificado. Resultados similares se encontraron en el estudio de Muratori et al. (2004), que encontró que los participantes que llevaban un año o menos con TCA mostraban una psicopatología menos grave y que la AN se asociaba con una alta tasa de psicopatología INT. En este sentido, diferenciaron tres tipos de AN adolescente en función de los perfiles de INT y EXT: 1) AN "normal" con un perfil no clínico; 2) AN INT "pura" con valores patológicos en las escalas de síndromes e INT (subtipo de AN restrictiva y/o atracones y purgas) y 3) AN "mixta" con valores patológicos en las escalas de síndromes, INT y EXT (subtipo de AN caracterizada por oposición, dificultades de concentración, donde los síntomas de INT son más graves).

En el estudio de Hodge et al. (2019) participaron adolescentes hospitalizadas que presentaban AN de tipo restrictivo. La muestra presentó un perfil interiorizado de problemas que se dividió en tres tipos distintos y que coinciden en la propuesta con los de Muratori et al. (2004). En la investigación de Forbush et al. (2010) el objetivo fue determinar dónde se encuadra el TCA en adolescentes dentro del modelo de INT y EXT. En el modelo mejor ajustado, los TCA formaron un subfactor del factor INT, en lugar de un subfactor del factor EXT, o su propia clase de trastorno, como sugiere el DSM. Los resultados del estudio indican que los TCA son una variante de los trastornos de INT. Forbush y Watson (2013) aplicaron un modelo ampliado de INT y EXT a una muestra representativa de hombres y mujeres. En este modelo los TCA formaron un subfactor dentro de la INT, al igual que se había encontrado en el estudio de Forbush et al. (2010) con población adolescente. Por otra parte, Mitchell et al. (2014) encontraron que las variables de AN, BN y TA se localizaban principalmente en el subfactor de angustia del dominio de INT más que con un factor de INT más general. Por último, el reciente estudio de Forbush et al. (2017) que propone desarrollar un modelo transdiagnóstico y jerárquico-dimensional para la psicopatología TCA que: 1) reduce la heterogeneidad diagnóstica, 2) incluye las dimensiones importantes de la 
psicopatología INT que a menudo se excluyen de los modelos de diagnóstico de TCA y 3) predice el deterioro clínico. Los niveles más bajos del modelo se caracterizan por factores que constituyen aspectos específicos de los TCA, de los trastornos afectivos y de ansiedad.

Según los estudios mencionados, parece que los TCA se asocian más frecuentemente con comportamientos INT, caracterizados por el afecto negativo, la preocupación y la rumiación. Tomados en conjunto, los resultados subrayan la importancia del papel que la sintomatología depresiva y la regulación emocional pueden desempeñar en el desarrollo y mantenimiento del TCA (Mitchell et al., 2014).

Por ello, esta investigación aporta información en cuanto a si los comportamientos exteriorizados o interiorizados son un elemento que puede estar presente en el inicio de los TCA durante adolescencia. Esto es interesante, ya que desde un modelo biopsicosocial de la psicopatología, los factores interiorizados y exteriorizados se entienden como factores inespecíficos de vulnerabilidad o riesgo en un amplio rango de psicopatologías (Kendler, Prescott, Myers y Neale, 2003). Los objetivos principales de este estudio: a) examinar si los comportamientos INT y EXT son un factor de riesgo proximal general o específico de la psicopatología en los TCA a su inicio y b) discriminar qué componentes sintomatológicos de la patología alimentaria son los que conforman un perfil INT o EXT en TCA.

Tras la revisión sistemática de Jacobi et al. (2004), donde concluyen la importancia del uso de diseños caso-control para estudiar los factores de riesgo en TCA al inicio de la patología. Siguiendo las recomendaciones, se han utilizado tres grupos control, uno con trastornos depresivos y otro con asma, y un grupo sin trastorno psiquiátrico. El grupo con trastorno afectivo nos ha permitido comparar con un grupo con grandes similitudes a nivel de correlatos psicológicos, así como síntomas (Ferreiro, Seoane y Senra, 2011) y consecuencias sociales, familiares y otros (Mischoulon et al., 2011). Ambas psicopatologías se ubican en la dimensión de INT (Adambegan et al., 2012; Forbush et al., 2017; Forbush et al., 2010; Forbush y Watson, 2013; Hodge et al. (2019); McDermott et al., 2006; Mitchell et al., 2014; Muratori et al., 2004). Comparten algunos factores relacionados con el temperamento (afectividad negativa) y la personalidad (alto neuroticismo e introversión) (Del Barrio, 2014). El grupo con patología asmática nos ha permitido comparar con un grupo con similitudes a nivel de impacto familiar, como limitaciones en la actividad diaria de las pacientes y sus familias, así como dependencia y atención excesiva sobre el síntoma (Theodoratou-Bekou et al., 2012) y de exacerbación sintomatológica debido al estrés (Yamamoto \& Nagano, 2015). De este modo, se ha encontrado un impacto en la calidad de vida y en el impacto emocional de la experiencia del cuidado en asma (Crespo, Carona, Silva, Canavarro y Dattilio, 2011), similar al que se ha encontrado en las familias con TCA (Anastasiadou Medina-Pradas, Sepúlveda y Treasure, 2014; Martín, Padierna, Aguirre, Quintana, Hayas y Muñoz, 2011). El grupo control sin patología nos ha permitido conocer qué correlatos podrían estar más relacionados con la adolescencia. Durante la adolescencia se pueden producir ciertos comportamientos desadaptativos que son solo variaciones de intensidad, respecto a las 
características de este periodo, más que una conducta psicopatológica (Achenbach y Rescorla, 2001).

Asimismo, los objetivos específicos de este estudio son los siguientes: 1) examinar las diferencias entre el grupo de TCA y los tres grupos control en los síndromes empíricos del "Listado de comportamientos para niños" (Child Behaviour Checklist, CBCL; Achenbach y Rescorla, 2001) y las dimensiones INT y EXT; 2) examinar las diferencias entre el grupo de TCA y los tres grupos control en los niveles patológicos de las dimensiones INT y EXT; 3) examinar la asociación en la muestra de TCA entre las dimensiones INT y EXT y las variables clínicas: patología alimentaria, depresión, ansiedad, sintomatología obsesivo-compulsiva y perfeccionismo; y 4) examinar la asociación en la muestra de TCA entre la patología alimentaria y los síndromes empíricos del CBCL.

\section{Método}

\section{Participantes}

La muestra estuvo compuesta por 180 mujeres adolescentes con edades comprendidas entre los 12-17 años y sus respectivas familias. La composición de la muestra fue de 50 pacientes con TCA, 40 pacientes con trastorno depresivo (TD), 40 pacientes con asma y 50 controles sanas. Todas las participantes fueron seleccionadas según los siguientes criterios de inclusión generales: ser mujer, tener entre 12 y 17 años de edad, de raza blanca y hablar correctamente español. Los criterios de exclusión fueron: la presencia de otras enfermedades que alteren el índice de masa corporal, presentar problemas del neurodesarrollo o patología psicótica. Además de estos criterios cada grupo presenta sus propios criterios específicos de inclusión y exclusión.

La muestra de mujeres con TCA fueron recogidas desde la Unidad de TCA del Hospital Universitario Infantil Niño Jesús durante el 2010-2012, presentaban síntomas de no más de un año de duración, no habían sido diagnosticadas ni habían recibido tratamiento previo. No debían tener un trastorno depresivo o historia de asma.

La muestra de mujeres con TD fue recogida con la colaboración del Hospital Sureste (CS. Arganda) (66,66\%) y el Hospital de Colmenar Viejo (33,33\%), entre 2014-2016. Los síntomas depresivos debían tener entre 12-24 meses de duración, aunque no habían sido diagnosticadas con TD. No podían tener un diagnóstico anterior o actual de TCA, ni presentar un índice de masa corporal (IMC) por debajo de 18 y por encima de 30 .

La muestra de mujeres con asma tenía este diagnóstico y se encontraba en seguimiento por el Servicio de Neumología del Hospital Universitario Niño Jesús durante al menos cuatro años. Los datos fueron recogidos entre 2014-2016. El diagnóstico de asma podía ser de tipo episódico frecuente, moderado o grave, de duración 3-6 años, haber sufrido hospitalizaciones y urgencias durante la infancia debidas a crisis de asma y haber padecido síntomas de asma durante el periodo escolar (6-12 años). Se excluyó a adolescentes con un diagnóstico anterior o actual de TCA o de un TD, presentar un IMC por debajo de 18 y por encima de 30. 
Finalmente, la muestra del grupo de control sano (sin patología psiquiátrica o de asma), recogida entre 2012-2015 de los institutos de secundaria de la Comunidad de Madrid, no presentaba en el presente ni en el pasado TCA o cualquier otro trastorno psiquiátrico según la entrevista diagnóstica, ni asma. Se excluyó a adolescentes con un IMC por debajo de 18 y por encima de 30.

\section{Instrumentos}

a) "Entrevista para trastornos afectivos y esquizofrenia en niños y adolescentes, versión para el presente y a lo largo de la vida" (Kiddie-Schedule for Affective Disorders and Schizophrenia, Present and Lifetime Version, K-SADS-PL; Kaufman, Birmaher, Brent, Rao y Ryan, 1996), versión en español de De la Peña et al. (2002). Se trata de una entrevista diagnóstica semiestructurada para los trastornos del eje I según los criterios del DSM-IV-TR, y adaptada en el presente estudio a los criterios del DSM-5 (APA, 2013). Se pregunta por dos o tres síntomas claves de cada trastorno y si sale positivo se pasa el suplemento para confirmar el diagnóstico. Las respuestas de los informantes se recogen de forma independiente (adolescente y madre), y si no queda claro, de forma conjunta. La entrevista cuenta con propiedades psicométricas adecuadas, oscilando la fiabilidad interjueces entre 0,76 y 1,0 (Ulloa et al., 2006). Las categorías en este estudio se dividieron en dos: $1=$ "presenta un diagnóstico clínico" y $0=$ "ausente".

b) "Listado de comportamientos para niños" (Child Behaviour Checklist, CBCL/618; Achenbach y Rescorla, 2001), versión en español de Sardinero, Pedreira y Muñiz (1997). El CBCL es un instrumento contestado por los padres para evaluar la presencia de psicopatología y/o problemas de comportamiento de niños y adolescentes durante los últimos seis meses. Está compuesto por 120 ítems, con tres alternativas de respuesta (0-2). Las opciones de respuesta son: "no es cierto (que sepa usted)", "algo, algunas veces es cierto", "cierto muy a menudo o bastante a menudo". La estructura factorial del cuestionario está compuesta por dos escalas de banda ancha (INT y EXT) y ocho escalas de banda estrecha que representan síndromes empíricos y que se encuadran dentro de la INT o EXT. Dentro de la INT se encuentran los siguientes síndromes empíricos: 1) el síndrome ansiedad/depresión, que evalúa sintomatología ansiosa y depresiva (p. ej., "llora mucho", "cree que tiene que ser perfecto", "demasiado ansioso/miedoso", "se siente culpable por cualquier cosa"); 2) el síndrome de aislamiento/depresión, que evalúa sintomatología depresiva en la que la persona se aísla de su entorno (p. ej., "se calla todo", "poco activo, lento, falta de energía") y 3) el síndrome de quejas somáticas, que evalúa problemas físicos sin causa aparente como dolores de cabeza, náuseas, problemas en la piel, vómitos, estreñimiento, entre otros. Dentro de la EXT están: 1) el síndrome de conducta anormativa, que evalúa conductas de quebrantar las normas (p. ej., "se salta las normas en casa, en la escuela y otros lugares", "va con jóvenes que se meten en problemas", "toma alcohol o drogas") y 2) el síndrome de conducta agresiva, que evalúa conductas de este tipo (p. ej., "es agresivo, cruel o malo con los 
demás", "se mete en muchas peleas"). Los otros síndromes empíricos que no se engloban dentro de las anteriores escalas de banda ancha son: 1) el síndrome de problemas sociales, que evalúa problemas de relación social ( $p$. ej., "se queja de que se siente sola", "los demás se burlan de ella a menudo"); 2) el síndrome problemas de pensamiento, que evalúa obsesiones, autoagresión/suicidio, problemas de sueño, conductas repetitivas (p. ej., "oye sonidos o voces que no existen", "movimientos nerviosos o tics", "ideas raras") y 3) el síndrome problemas de atención, que evalúa la falta de atención, hiperactividad e impulsividad (p. ej., "no puede concentrarse o prestar atención durante mucho tiempo", "no puede estar quieta sentada", "actúa sin pensar"). Las puntuaciones del cuestionario se pueden convertir en puntuaciones $T(M=50 ; D T=10)$ para establecer categorías cualitativas que reflejan la gravedad de los síntomas. Las puntuaciones "patológicas" o "clínicamente relevantes" están definidas por puntuaciones $T \geq 64$ en las escalas de banda ancha y $\geq 70$ en las escalas de síndromes empíricos. Los valores de alfa de Cronbach están entre 0,72 y 0,91 en la validación original. En la muestra actual, para las escalas de banda ancha INT y EXT los coeficientes alfa de Cronbach fueron de 0,85 y 0,86, respectivamente.

c) "Inventario de trastornos de la conducta alimentaria" (Eating Disorder Inventory-2, EDI-2; Garner, 1991), versión en español de Corral, González, Pereña y Seisdedos (1998). Se trata de un inventario de 91 ítems con una escala Likert de seis puntos (de $0=$ nunca a $5=$ siempre). Consta de 11 escalas y se utilizaron las ocho dimensiones principales: tres que evalúan actitud y conductas relacionadas con la comida, el peso y la forma física (Obsesión por la delgadez, Bulimia, Insatisfacción corporal) y cinco dimensiones generales (Ineficacia, Perfeccionismo, Desconfianza interpersonal, Conciencia interoceptiva y Miedo a la madurez). A mayor puntuación mayor nivel de sintomatología alimentaria, mayor nivel de obsesión por la delgadez o mayor insatisfacción corporal. La consistencia interna de las distintas subescalas en la adaptación española varía de 0,82 a 0,92, excepto en la subescala Miedo a la madurez que fue de 0,65. En esta muestra fue de 0,80 a 0,94 para las distintas subescalas.

d) "Inventario de depresión infantil" (Children's Depression Inventory, CDI; Kovacs, 1992), versión en español de Del Barrio y Carrasco (2004). El CDI evalúa sintomatología depresiva mediante 27 ítems, que se puntúan de 0 a 2 según la intensidad ( $0=$ "normalidad", $1=$ "cierta intensidad del síntoma no excesiva" y 2= síntoma propio de la depresión"). El rango de puntuaciones está entre 0 y 54. A mayor puntuación mayor nivel de sintomatología depresiva. En muestras clínicas, los valores de alfa de Cronbach variaban entre 0,71 y 0,89 . En nuestra muestra actual, el alfa de Cronbach fue de 0,88.

e) "Inventario de ansiedad estado-rasgo para niños" (State-Trait Anxiety Inventory for Children, STAIC; Spielberger, Edwards, Lushene, Montuori y Platzek, 1973), versión en español de Seisdedos (1990). El STAIC evalúa la ansiedad como estado (AVE) referido a una ansiedad del momento actual y como rasgo $(A / R)$, referido a una ansiedad disposicional, estable, que tiende a evaluar las situaciones como amenazadoras. Cada escala tiene 20 ítems, con 
un rango de respuesta de 1-3. En la parte A/E las opciones de respuesta son: "nada", "algo", "mucho"; y en el A/R son: "casi nunca", "a veces", "a menudo". El rango de puntuaciones para cada escala es de 20-60. A mayor puntuación mayor nivel de ansiedad-estado y de ansiedad-rasgo. La consistencia interna en la validación española fue de 0,89 para la subescala A/E y de 0,85 para la subescala de A/R. En la muestra actual fue de 0,86 para la A/E y 0,92 para la A/R.

f) "Inventario obsesivo de Leyton, versión para niños" (Leyton Obsessional Inventory-Child Version, LOI-CV; Berg, Rapoport y Flament, 1986). Se trata de un inventario de 20 ítems que evalúa la ausencia o presencia de preocupaciones o conductas obsesivas (puntuaciones 0-1, no o sí) y el rango teórico de puntuaciones es de 0-20. A mayor puntuación mayor nivel de sintomatología obsesiva. El alfa de Cronbach informado fue de 0,79 para la presencia de síntomas y de 0,90 para la interferencia. En esta muestra fue de 0,82 para la presencia de síntomas y de 0,89 para la interferencia.

g) "Escala de perfeccionismo para niños y adolescentes" (Child and Adolescent Perfectionism Scale, CAPS-22; Flett et al., 2000), versión en español de Castro et al. (2004). El CAPS-22 evalúa perfeccionismo y está formado por 22 ítems que se responden en una escala Likert (1-5). Las opciones de respuesta son: "falso, no me sucede nunca", "bastante falso", "ni cierto ni falto", "bastante cierto", "muy cierto en mi caso". Se compone de dos escalas: perfeccionismo autoorientado (12 ítems, puntuaciones entre 12-60) y perfeccionismo preescrito socialmente (10 ítems, puntuaciones entre 10-50). A mayor puntuación mayor perfeccionismo. La consistencia interna en la validación española fue de 0,89 y en la muestra actual fue de 0,92.

\section{Procedimiento}

Se trata de un estudio caso-control, donde las participantes de cada grupo control han sido emparejadas a cada una al grupo TCA (1:1), por edad y nivel socioeconómico y educativo de los padres. Para el cálculo del nivel socioeconómico se utilizó el índice de la "Escala de Hollingshead Redlich" (Hollingshead Redlich Scale; Hollingshead y Redlich, 1953). Este índice se calcula para cada uno de los padres y determina dicho estatus teniendo en cuenta el nivel educativo y la ocupación laboral, dando como resultado un estatus que puede ir del I (muy bajo) al V (muy alto). Para el emparejamiento se tuvo en cuenta el estatus más alto de entre los dos padres. Se detalla más exhaustivamente la selección de las muestras en otro estudio publicado (Sepúlveda et al., en prensa). La investigación contó con el consentimiento de la Comisión de Investigación del Hospital Universitario Niño Jesús (Ref. 0009/10) y del Comité Ético de la Universidad Autónoma de Madrid (CEI-27-673).

La recogida de datos se llevó a cabo entre el 2012 y el 2016. En primer lugar, se recogió a la muestra con TCA en el hospital infanto-juvenil y a partir de las características de esta muestra se recogió la muestra sin patología. Después, se recogió en paralelo la muestra con patología afectiva y con patología asmática, emparejados a los casos TCA (1:1), si cumplían los criterios establecidos. Los 
profesionales de las distintas unidades de referencia fueron los responsables de reclutar a los participantes. Tras esto, se realizaron llamadas telefónicas para valorar las variables sociodemográficas, personales y clínicas, con el objetivo de valorar si cumplían los criterios de inclusión del estudio y emparejar. En segundo lugar, tuvo lugar la fase de evaluación, realizada en los centros de referencia (hospital, centro de salud o centro educativo). Al comienzo de la evaluación se explicaba y se firmada el consentimiento informado por la participante y su familiar, tras esto se realizaba una entrevista para evaluar la historia clínica y la entrevista semiestructurada. La duración aproximada de la evaluación era de dos horas. Al finalizar, se entregaba una batería de cuestionarios para las participantes y otra para cada uno de los progenitores, que tenían que completar. En tercer lugar, se entrega un informe psicológico a la participante/familia. Si era necesario se entrega una hoja de derivación clínica a un centro de referencia.

\section{Análisis de datos}

La normalidad fue evaluada a través de la prueba a de Kolmogorov-Smirnov, como la muestra no cumplió con una distribución normal, se realizaron pruebas no paramétricas. Para el análisis descriptivo de las variables cuantitativas se calcularon la media y la desviación típica, mientras que las variables cualitativas fueron descritas mediante frecuencias. Para comprobar las diferencias en las variables continuas entre el TCA y los distintos grupos controles se realizaron la prueba Kruskal-Wallis para comparaciones de más de dos grupos y, para las comparaciones entre dos grupos, la prueba de Mann-Whitney ajustando mediante la prueba de Bonferroni para reducir el riesgo de cometer error tipo I en cada uno de los contrastes realizados. Para las comparaciones entre variables categóricas se realizó la prueba $\chi^{2}$ de Pearson, en los casos en que se realizó una comparación con dos categorías en cada variable se ha incluido la correlación por continuidad de Yate para compensar la sobreestimación del valor de $\chi^{2}$; además en aquellas comparaciones en que no se cumplía la frecuencia mínima de 5 en una de las variables se ha utilizado el estadístico de Fisher. Para conocer la asociación entre las variables psicológicas objeto de estudio en la muestra de TCA se ha calculado el coeficiente de correlación rho de Spearman.

Para los análisis estadísticos se utilizó el programa IBM SPSS Statistics v. 19.0. Todos los valores de $p$ fueron bilaterales y la significación estadística se estableció en $p<0,05$, salvo en las comparaciones múltiples que se estableció la corrección por Bonferroni.

\section{Resultados}

En la tabla 1 se presentan las características sociodemográficas de las cuatro muestras de adolescentes y sus familias. No se encontraron diferencias significativas entre la edad y el estatus socioeconómico de la muestra. En cuanto a los diagnósticos recogidos por la entrevista K-SADS y adaptados a los criterios diagnósticos del DSM-5 (APA, 2013) se encuentra lo siguiente: En el grupo de TCA un $70 \%(n=35)$ cumple el diagnóstico de AN subtipo restrictivo, un $16 \%$ 
$(n=8)$ el diagnóstico AN purgativa y el $14 \%(n=7)$ otro trastorno alimentario o de la ingestión de alimentos no especificado. En el grupo de TD, un $80 \%(n=32)$ cumple el diagnóstico de trastorno de depresión mayor, un 7,5\% (n=3) el diagnóstico de trastorno depresivo persistente (Distimia), un 10\% ( $n=4)$ con diagnóstico de trastorno depresivo no especificado y un $2,5 \%(n=1)$, trastorno de desregulación perturbador del estado de ánimo.

Tabla 1

Características sociodemográficas y comparación entre las cuatro muestras

\begin{tabular}{|c|c|c|c|c|c|}
\hline \multirow{2}{*}{ Características } & $\begin{array}{c}\text { TCA } \\
(n=50)\end{array}$ & $\begin{array}{c}\text { TD } \\
(n=40)\end{array}$ & $\begin{array}{c}\text { AS } \\
(n=40)\end{array}$ & $\begin{array}{c}C S \\
(n=50)\end{array}$ & $p$ \\
\hline & $M(D T)$ & $M(D T)$ & $M(D T)$ & $M(D T)$ & \\
\hline Edad (años) & $14,68(1,39)$ & $15,10(1,55)$ & $14,73(1,74)$ & $14,66(1,31)$ & $0,435^{a}$ \\
\hline $\begin{array}{l}\text { Tiempo de tratamiento } \\
\text { previo (meses) }\end{array}$ & $3,30(4,43)$ & $8,23(5,61)$ & -- & -- & $0,001 * * b$ \\
\hline $\begin{array}{l}\text { Duración del trastorno } \\
\text { (meses) }\end{array}$ & $10,32(7,41)$ & -- & -- & -- & \\
\hline IMC $\left(\mathrm{kg} / \mathrm{m}^{2}\right)$ & $16,07(1,74)$ & $22,41(2,95)$ & $21,33(2,86)$ & $21,16(2,67)$ & $0,001 * * a$ \\
\hline Tratamiento actual & $n(\%)$ & $n(\%)$ & $n(\%)$ & $n(\%)$ & \\
\hline Ingreso hospitalario & $36(72,0)$ & $4(10,3)$ & & & \\
\hline $\begin{array}{l}\text { Hospitalización } \\
\text { domiciliaria }\end{array}$ & $12(24,0)$ & & & & \\
\hline $\begin{array}{l}\text { Seguimiento } \\
\text { ambulatorio }\end{array}$ & $2(4,0)$ & $25(64,1)$ & $40(100,0)$ & & \\
\hline No recibe tratamiento & & $1(2,6)$ & & $50(100,0)$ & \\
\hline Valores perdidos & & $9(23,1)$ & & & \\
\hline \multicolumn{6}{|l|}{ Datos familias } \\
\hline \multicolumn{6}{|l|}{ Estatus socioeconómico } \\
\hline I (bajo) & $6(12,0)$ & $4(8,0)$ & $2(4,0)$ & $4(8,0)$ & $0,145^{c}$ \\
\hline II (medio-bajo) & $4(8,0)$ & $7(14,0)$ & $6(12,0)$ & $3(6,0)$ & \\
\hline III (medio) & $6(12,0)$ & $10(20,0)$ & $6(16,0)$ & $7(14,0)$ & \\
\hline IV (medio-alto) & $10(20,0)$ & $11(22,0)$ & $13(26,0)$ & $11(22,0)$ & \\
\hline $\mathrm{V}$ (alto) & $24(48,0)$ & $8(16,0)$ & $11(22,0)$ & $25(50,0)$ & \\
\hline \multicolumn{6}{|l|}{ Tipo de familia } \\
\hline Nuclear & $38(76,0)$ & $24(48,0)$ & $35(70,0)$ & $39(79,6)$ & $0,031 * c$ \\
\hline Monoparental & $12(24,0)$ & $16(32,0)$ & $5(10,0)$ & $10(20,4)$ & \\
\hline
\end{tabular}

Notas: $\mathrm{TCA}=$ trastorno del comportamiento alimentario; $\mathrm{TD}=$ trastornos depresivos; $\mathrm{AS}=$ asma; $\mathrm{CS}=$ control sano; $I M C=$ índice de masa corporal. ${ }^{a}$ Prueba de Kruskal-Wallis; ${ }^{b}$ prueba de Mann-Whitney; cprueba de chi-cuadrado. ${ }^{*} p<0,05 ;{ }^{*} p<0,01$.

Respecto a la comorbilidad, un $22 \%$ de las participantes del grupo con TCA presentaban comorbilidad: un $18 \%(n=9)$ con trastorno de ansiedad y un $4 \%$ $(n=2)$ con trastorno obsesivo compulsivo (TOC). En el grupo con trastorno afectivo se identificó comorbilidad en el $25 \%$ de los casos: un 2,5\% $(n=1)$ con trastorno esquizofectivo, un $12,5 \%(n=5)$ con trastorno de ansiedad, un $2,5 \%$ $(n=1)$ con enuresis, un $2,5 \%(n=1)$ con TDAH y un $5 \%(n=2)$ con abuso de sustancias. Por su parte, un $17,5 \%$ de las participantes del grupo con patología 
asmática presentó comorbilidad: un 5\% $(n=2)$ con trastorno de ansiedad, un 5\% $(n=2)$ con trastorno adaptativo, un $2,5 \%(n=1)$ con TOC y un $5 \%(n=2)$ con TDAH. Por último, en el grupo sin patología se encontró comorbilidad en el $6 \%$ de los casos: un $2 \%(n=1)$ con distimia, un $2 \%(n=1)$ con TOC y un $2 \%(n=1)$ con trastorno de ansiedad.

La tabla 2 refleja los resultados de la prueba de Kruskal-Wallis para examinar si existen diferencias en las distintas variables del CBCL entre las cuatro muestras. Se encontraron diferencias significativas entre los cuatros grupos en algunas variables del $C B C L$ del padre: ansiedad/depresión, aislamiento/depresión, quejas somáticas, problemas de pensamiento, problemas de atención, INT y EXT. Tras la prueba de Mann-Whitney se observa que en las variables de ansiedad/depresión, aislamiento/depresión, problemas de pensamiento, problemas de atención, e INT y EXT sólo se encuentran diferencias significativas con los grupos de AS y CS. Y, en la variable de quejas somáticas sólo se encuentran diferencias con el grupo CS. A diferencia de esto, la prueba de Kruskal-Wallis revela diferencias significativas entre los cuatros grupos en todas las variables del CBCL de la madre. Tras la prueba de Mann-Whitney y se observa que en las variables de ansiedad/depresión, aislamiento/depresión, problemas de pensamiento e INT sólo se encuentran diferencias significativas con los grupos de AS y CS. En las variables de quejas somáticas y problemas sociales sólo se encuentras diferencias con el grupo CS. Por otra parte, en la variable de problemas de atención se encuentran diferencias sólo con el grupo de TD, y en la variable de EXT en el grupo de AS.

En las figuras 1 y 2 se encuentra un resumen gráfico de la prueba $\chi^{2}$ de Pearson sobre la independencia de proporciones para comparar las puntuaciones patológicas en las variables de INT y EXT entre el grupo de TCA y el resto de grupos control. Se obtuvieron diferencias significativas en la variable de INT del padre y la madre con los grupos de AS y CS.

En la tabla 3 se calculó el coeficiente correlación de Spearman para estudiar la relación entre las dimensiones de INT y EXT descrita por ambos progenitores y las variables clínicas (patología alimentaria, depresión, ansiedad, rasgos obsesivoscompulsivos y perfeccionismo). En la INT puntuada por el padre destaca la relación positiva y significativa con la puntuación total del EDI-2, así como de sus subescalas obsesión por la delgadez, ineficacia y conciencia interoceptiva. También la INT se relaciona positiva y significativamente con la depresión, la ansiedad rasgo y la sintomatología obsesivo compulsiva.

Por otra parte, la INT descrita por la madre se asocia positiva y significativamente con la puntuación total del EDI-2, así como de sus subescalas obsesión por la delgadez, insatisfacción corporal, ineficacia, desconfianza interpersonal y conciencia interoceptiva. También la INT se relaciona positiva y significativamente con la depresión, la ansiedad rasgo y la sintomatología obsesivo compulsiva. 


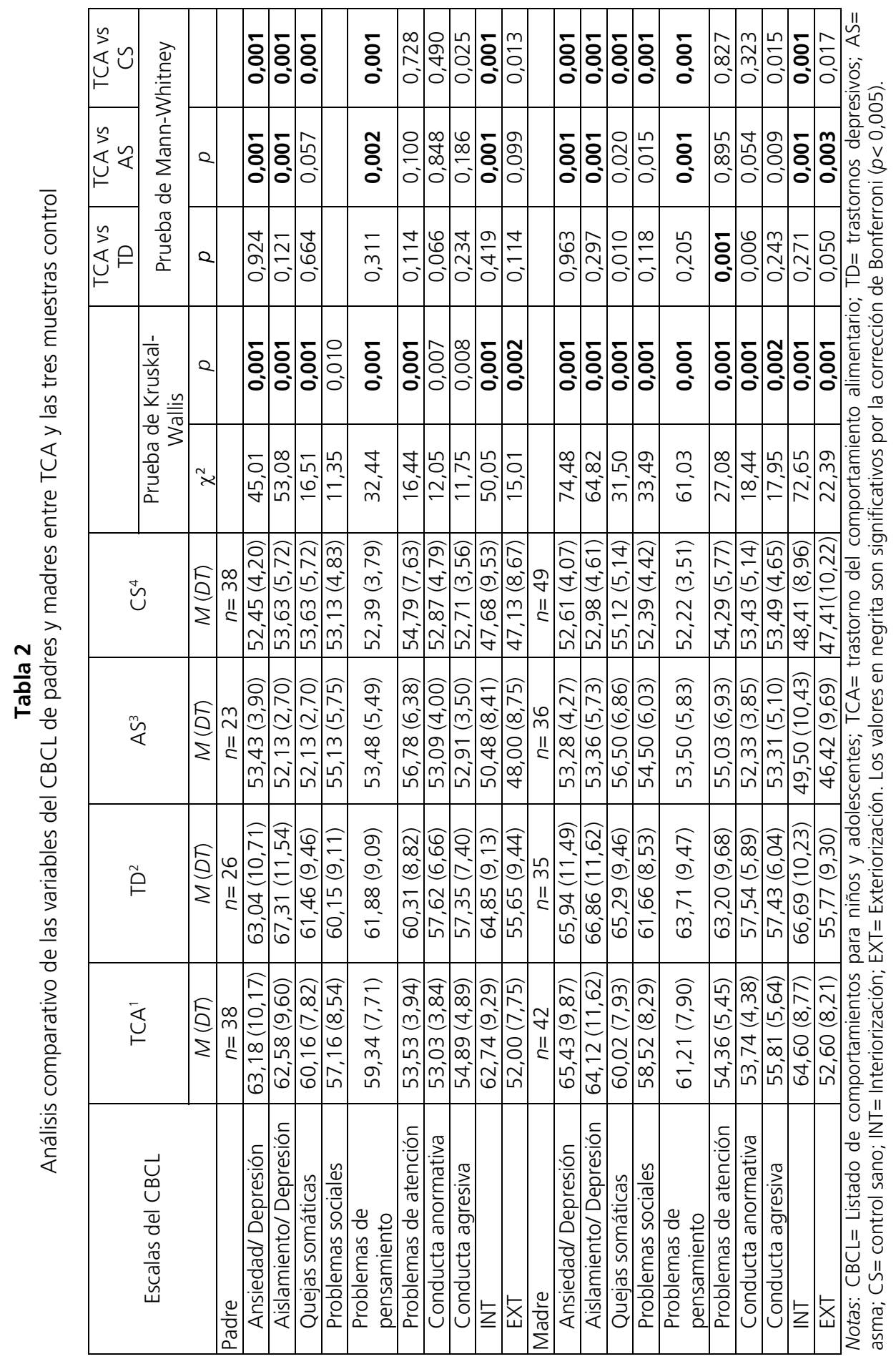




\section{Figura 1}

Diferencias en la proporción de puntuaciones patológicas en la INT y EXT de los padres entre el TCA versus los tres grupos control en el CBCL-padre

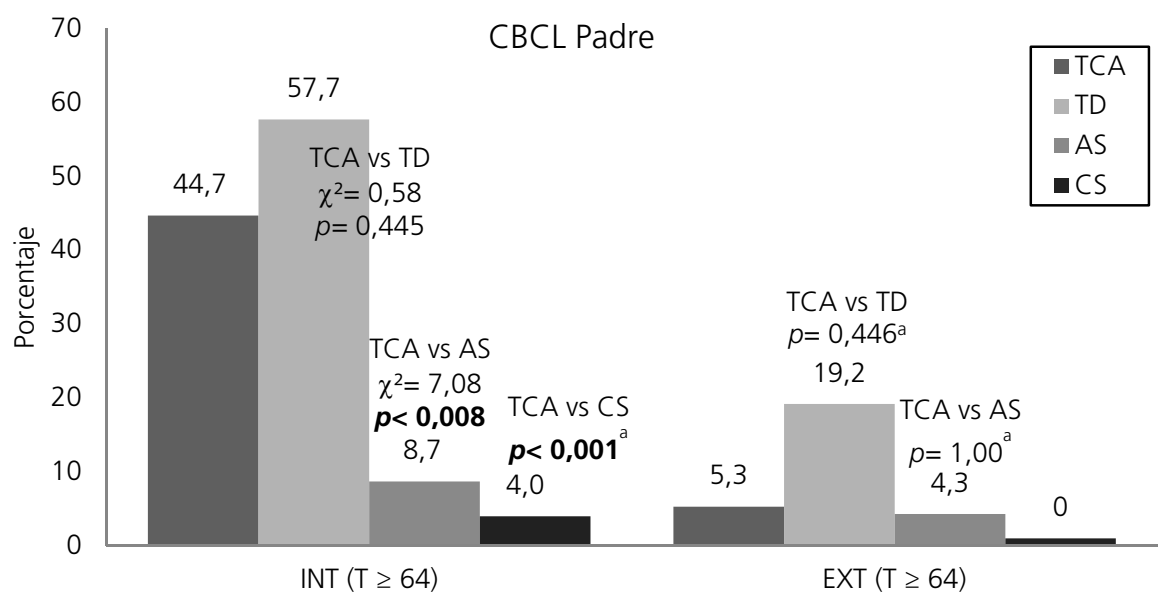

Notas: $\mathrm{CBCL}=$ Listado de comportamientos infantiles; $\mathrm{TCA}=$ trastorno del comportamiento alimentario; $\mathrm{TD}=$ trastornos depresivos; $\mathrm{AS}=$ asma; $\mathrm{CS}=$ control sano; $\mathrm{INT}=$ interiorización; EXT= exteriorización. $\chi^{2}$ con corrección por continuidad de Yate. Puntuación patológica $T \geq 64$. ${ }^{a} E n$ aquellas comparaciones en que no se cumple una frecuencia mínima en una de las variables de 5 se utiliza el estadístico de Fisher. Los valores en negrita son significativos $(p<0,05)$.

\section{Figura 2}

Diferencias en la proporción de puntuaciones patológicas en la INT y EXT de las madres entre el TCA versus los tres grupos control en el CBCL-madre

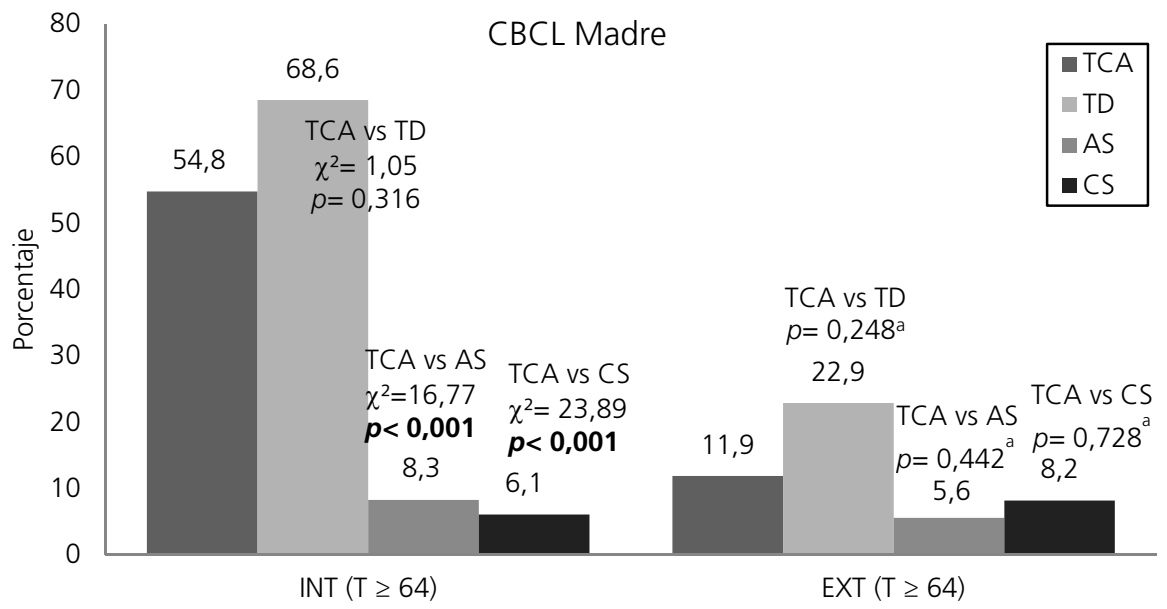

Notas: $\mathrm{CBCL}=$ Listado de comportamientos para niño; $\mathrm{TCA}=$ trastorno del comportamiento alimentario; $\mathrm{TD}=$ trastornos depresivos; $\mathrm{AS}=$ asma; $\mathrm{CS}=$ control sano; $\mathrm{INT}=$ interiorización; $\mathrm{EXT}=$ exteriorización. $\chi^{2}$ con corrección por continuidad de Yate. Puntuación patológica $T \geq 64$. ${ }^{a}$ En aquellas comparaciones en que no se cumple una frecuencia mínima en una de las variables de 5 se utiliza el estadístico de Fisher. Los valores en negrita son significativos $(p<0,050)$. 
Tabla 3

Correlaciones entre las dimensiones de INT y EXT y las variables clínicas en la muestra de TCA

\begin{tabular}{|l|c|c|c|c|}
\hline \multirow{2}{*}{ Variables clínicas } & \multicolumn{2}{|c|}{ Padres } & \multicolumn{2}{c|}{ Madres } \\
\cline { 2 - 5 } & INT & EXT & INT & EXT \\
\hline EDI-2 Total & $0,48^{* *}$ & 0,24 & $0,56^{* *}$ & 0,23 \\
\hline DELG & $0,46^{* *}$ & 0,14 & $0,45^{* *}$ & 0,19 \\
\hline BN & 0,04 & 0,23 & 0,22 & 0,29 \\
\hline INSA & 0,22 & 0,12 & $0,33^{*}$ & 0,04 \\
\hline INEF & $0,45^{* *}$ & 0,29 & $0,41^{* *}$ & 0,24 \\
\hline PERF & 0,31 & 0,28 & 0,31 & 0,25 \\
\hline DESC & 0,21 & 0,05 & $0,45^{* *}$ & 0,13 \\
\hline CONC & $0,48^{* *}$ & 0,23 & $0,47^{* *}$ & 0,29 \\
\hline MADU & 0,29 & 0,09 & 0,23 & 0,02 \\
\hline CDI & $0,45^{* *}$ & 0,11 & $0,50^{* *}$ & 0,25 \\
\hline STAIC-Estado & 0,29 & 0,15 & 0,31 & 0,28 \\
\hline STAIC-Rasgo & $0,48^{* *}$ & 0,23 & $0,56^{* *}$ & 0,30 \\
\hline LOI-CV & $0,39^{*}$ & 0,20 & $0,48^{* *}$ & 0,26 \\
\hline CAPS- AUTOPER & 0,24 & 0,15 & 0,21 & 0,14 \\
\hline CAPS- PERSOC & 0,14 & 0,14 & 0,11 & 0,16 \\
\hline
\end{tabular}

Notas: INT= Interiorización; EXT = Exteriorización; EDI-2= Inventario de trastornos de la conducta alimentaria; $\mathrm{DELG}=$ Obsesión por la delgadez; $\mathrm{BN}=$ Bulimia; INSA= Insatisfacción corporal; INEF= Ineficacia; $\mathrm{PERF}=$ Perfeccionismo; $\mathrm{DESC}=$ Desconfianza interpersonal; $\mathrm{CONC}=$ Conciencia interoceptiva; $\mathrm{MADU}=$ Miedo a la madurez; $\mathrm{CDI}=$ Inventario de depresión infantil; $\mathrm{STAIC}=$ Inventario de ansiedad estado-rasgo para niños; $\mathrm{LOI}-\mathrm{CV}=$ Inventario obsesivo de Leyton, versión para niños; CAPS= Escala de perfeccionismo para niños y adolescentes; $A U T O P E R=$ Autoperfeccionismo; $P E R S O C=$ Perfeccionismo percibido de forma social. ${ }^{*} p<0,05$ (bilateral); ${ }^{*} p<0,01$ (bilateral).

En tabla 4 se calculó el coeficiente correlación de Spearman para estudiar la relación entre la puntuación total del EDI-2 de la muestra de TCA y los síndromes empíricos del CBCL puntuados por ambos progenitores.

\section{Tabla 4}

Correlaciones entre los síndromes empíricos del CBCL y la puntuación total del EDI-2 de la muestra de TCA

\begin{tabular}{|l|c|c|}
\hline \multirow{2}{*}{ Síndromes empíricos del CBCL } & \multicolumn{2}{|c|}{ Puntuación total del EDI-2 } \\
\cline { 2 - 3 } & Padres & Madres \\
\hline Ansiedad/depresión & $0,45^{* *}$ & $0,51^{* *}$ \\
\hline Aislamiento/depresión & $0,36^{*}$ & $0,46^{* *}$ \\
\hline Quejas somáticas & $0,37^{*}$ & $0,43^{* *}$ \\
\hline Problemas sociales & 0,32 & $0,33^{*}$ \\
\hline Problemas de pensamiento & $0,45^{* *}$ & $0,40^{*}$ \\
\hline Problemas de atención & $0,33^{*}$ & $0,39^{*}$ \\
\hline Conducta anormativa & 0,29 & 0,12 \\
\hline Conducta agresiva & 0,14 & 0,15 \\
\hline
\end{tabular}

Notas: $\mathrm{CBCL}=$ Listado de comportamientos de niños; $\mathrm{EDI}-2=$ Inventario de trastornos de la conducta alimentaria. ${ }^{*} p<0,05$ (bilateral); ${ }^{* *} p<0,01$ (bilateral). 
En los datos de los padres destaca la relación positiva y significativa entre la puntuación total del EDI-2 y los síndromes empíricos de ansiedad/depresión, aislamiento/depresión, quejas somáticas, problemas de pensamiento y problemas de atención. Por otra parte, en los datos de la madre destaca la relación positiva y significativa entre la puntuación total del EDI-2 y los síndromes empíricos de ansiedad/depresión, aislamiento/depresión, quejas somáticas, problemas sociales, problemas de pensamiento y problemas de atención.

\section{Discusión}

La INT es un factor de riesgo proximal general para la psicopatología general (TCA y TD), ya que los niveles de INT patológica sí presentaron diferencias significativas entre el grupo de TCA y los grupos control de asma y sanas, pero no con TD. Esto resultados apoyan evidencia empírica previa donde se establecía que tanto el TCA como el TD compartían la dimensión de INT (Forbush et al., 2010; Forbush y Watson, 2013; Hodge et al. 2019; Mitchell et al., 2014). Esto podría explicar la alta comorbilidad que existe entre ambos trastornos (McDermott et al., 2006) y la dificultad de realizar un diagnóstico diferencial en la adolescencia entre TCA y TD, pues ambas adolescentes tendrían una tendencia a interiorizar sus síntomas. Además, esta comorbilidad se presenta de igual forma independiente de la edad (Preti et al., 2009), lo que hace que en el inicio de la psicopatología sea difícil identificar a qué categoría diagnóstica específica corresponde. Sin embargo, esto también describe un hallazgo positivo de cara a la prevención de ambos trastornos. Si la INT de los problemas en la adolescencia es un factor de riesgo para el desarrollo de TCA y TD, esto ayudaría a identificar grupos de riesgo para presentar un problema psicopatológico y contribuiría a mejorar las estrategias de prevención o un objetivo en el tratamiento en una etapa temprana.

Por otra parte, los componentes sintomatológicos de las pacientes con TCA que se relacionan con la INT son: la obsesión por la delgadez, la ineficacia, la conciencia interoceptiva del EDI-2, la depresión, la ansiedad-rasgo y la sintomatología obsesivo-compulsiva. La relación entre la INT y las tres escalas del EDI-2, indica que es una forma en que la persona está dando una explicación interiorizada a los síntomas que presenta. La obsesión por la delgadez habla de la preocupación referida al peso, la realización de dietas y el miedo a engordar; la ineficacia son los sentimientos de incapacidad general, inseguridad, vacío, autodesprecio y falta de control sobre la propia viva; y la conciencia interoceptiva es el grado de confusión o dificultad para responder adecuadamente a los estados emocionales (Garner, 1991). La relación entre la INT y la depresión se explica porque es parte de la definición de la INT desde el modelo que lo sostiene, siendo una de las patologías claves relacionadas con esta variable (Achenbach y Rescorla, 2001; Cosgrove et al., 2011; Eaton et al., 2012). La relación entre la INT y la ansiedad-rasgo hace referencia a la ansiedad percibida usualmente por la persona (Spielberger et al., 1973), la INT mide la sintomatología de ansiedad durante los seis últimos meses y la ansiedad estado en la última semana, con lo cual el tiempo que mide la ansiedad podría ser lo que indique la relación entre ambas. 
Asimismo, la sintomatología obsesivo-compulsiva ha sido conceptualizada dentro de la INT en los estudios previos que la han evaluado (Mitchell et al., 2014). Por último, llama la atención, que aun siendo el perfeccionismo uno de los factores de riesgo más importante para la AN (Fairburn, Cooper, Doll y Welch, 1999; Jacobi et al., 2004; Machado, Gonçalves, Martins, Hoek y Machado, 2014; Pike et al., 2008) no se encuentre relacionado con la INT, pudiendo explicarse porque en el $\mathrm{CBCL}$ solo hay un ítem relacionado con el perfeccionismo "Cree que tiene que ser perfecto(a)" y resulta insuficiente para medir apropiadamente esta dimensión.

Por último, la patología alimentaria en TCA está asociada específicamente desde el modelo de Achenbach y Rescorla (2001) con la ansiedad/depresión, el aislamiento/depresión, las quejas somáticas, los problemas de pensamiento, los problemas de atención y la INT. La relación con el aislamiento/depresión confirma los múltiples resultados encontrados que han dado lugar al modelo de dificultades interpersonales de AN, de Treasure y Schmidt (2013), relacionadas con la tendencia recurrente de evitar el conflicto o sus propias reacciones emocionales y disminuye sus habilidades de afrontamiento ante la adversidad real o percibida. De hecho, se ha encontrado que los adolescentes que perciben sus emociones de forma inadecuada presentan un mayor desajuste clínico y emocional y un peor ajuste personal (Palomera, Salguero y Ruiz-Aranda, 2012). La relación con las quejas somáticas puede tener una doble explicación, una causa física ante la renutrición en la primera fase del tratamiento (como son dolores o molestias en el estómago o retortijones, náuseas, encontrarse mal, vómitos), o la continuación del malestar emocional que siente la adolescente que se transmite a través de estos síntomas físicos (APA, 2013). Por otra parte, los resultados no esperados con la asociación con problemas de pensamiento y problemas de atención. El primero se puede relacionar con la propia alteración en las actitudes y conducta propias de sintomatología del TCA, que se recogen en los ítems del $\mathrm{CBCL}$ sobre autoagresiones, problemas de sueño, pellizcarse partes del cuerpo, repetir determinadas acciones o tener ideas y conductas extrañas, muy relacionadas estas últimas con los rasgos obsesivos-compulsivos (Treasure y Schmidt, 2013). La relación entre la patología alimentaria y los problemas de atención se explican en sus dos vertientes, existe una disminución de la atención, al presentar rumiaciones recurrentes relacionadas con contar calorías, el peso y/o la imagen corporal, y esto refleja que los padres observen una inversión mayor de tiempo para estudiar, más ariscas, menos colaborativas, aunque esta desconexión conductual no suele reflejarse en una disminución del rendimiento escolar (Sepúlveda et al., en prensa). A su vez, estas adolescentes incrementan el número de actividades que realizan con el fin de influir en su peso y/o imagen corporal, unido a los efectos de la desnutrición (McDermott et al., 2006) y, por tanto, los padres perciben esta hiperactividad en sus hijas, y más prevalente al inicio de la patología (Meyer, Taranis, Goodwin y Haycraft, 2011).

En los resultados se han encontrado diferencias entre los $C B C L$ contestados por el padre y por la madre. Los resultados aportados por las madres en general han reportado más gravedad y se han destacado más sintomatología interiorizada que los padres. En la literatura se encuentra que en general hay más acuerdo para los problemas exteriorizados que interiorizados, y que son las madres las que 
informan más problemas de interiorización que los padres (Achenbach, McConaughy y Howell, 1987; Phares, 1997; Treutler y Epkins, 2003). Las madres suelen tener más contacto con el niño que los padres, por ello pueden ser más conscientes de los problemas de sus hijos o pueden sesgar más sus interpretaciones (Treutler y Epkins, 2003).

En base a los resultados del estudio se pueden identificar algunas ventajas y limitaciones del modelo dimensional frente al categorial. Como ventaja, los modelos dimensionales son capaces de explicar mejor la co-ocurrencia y la escasa estabilidad diagnóstica (Forbush et al., 2010); y también ayuda a que la multiplicación de categorías diagnósticas actuales se revierta en el futuro, ya que los trastornos son definidos en términos dimensionales (Lemos, 2003). Como desventaja, al final los factores de INT y EXT son factores inespecíficos de vulnerabilidad o riesgo para un amplio rango de trastornos y síntomas (Kendler et al., 2003) y no todas tienen la misma gravedad clínica. Además, en los estudios dimensionales aún quedan muchas psicopatologías por incluir, por ejemplo, los TCA, que en comparación con otras psicopatologías han sido poco estudiados desde esta perspectiva (Forbush et al., 2010; Forbush y Watson, 2013; Mitchell et al., 2014). Por ello, de momento sería recomendable que ambas perspectivas convivan y se puedan llegar a aplicar modelos mixtos de psicopatología.

El presente estudio tiene también una serie de limitaciones: a) no se han tomado medidas de exteriorización como podrían ser la impulsividad, agresividad o hiperactividad de la muestra; b) no se ha aplicado el "Autoinforme para el adolescente" (Youth Self-Report, YSR) del protocolo multiaxial ASEBA (lo que podría haber dado información contrastada sobre la INT y EXT evaluada por las propias adolescentes; c) es posible que en algún caso, los resultados encontrados respecto a la interiorización puedan tener que ver con la comorbilidad encontrada con los trastornos de ansiedad; d) la muestra estaba compuesta exclusivamente por mujeres adolescentes dado que es un importante factor de riesgo (Jacobi et al., 2004). Sin embargo, en la literatura sobre el modelo de INT y EXT en TCA se sugiere que los TCA están influenciados por las mismas variables psicopatológicas tanto en hombres como mujeres, con lo que las conclusiones de este estudio se podrían ampliar a varones (Mitchell et al., 2014). Y, otras posibles líneas de continuidad del presente trabajo pueden ser: a) ampliar la muestra de TCA, tratando de incluir otros diagnósticos como el de BN o TA; b) continuar el estudio de forma longitudinal para estudiar si los valores de INT y EXT pueden predecir la migración, o no, de una sintomatología relacionada con el TCA, tipo restricción, purga, atracón, etc a otra distinta, así como a la comorbilidad categorial con otro diagnóstico; c) abordar si la INT puede actuar como mediador o moderador de otros factores de riesgo estudiados en TCA.

La INT de los problemas en la adolescencia puede ser un factor de riesgo para el desarrollo de TCA, lo que puede ayudar a identificar los grupos de riesgo y contribuir a mejores estrategias de prevención o un objetivo en el tratamiento en una etapa temprana del trastorno (Adambegan et al., 2012). En la revisión de Jacobi et al. (2004) se encuentra que los programas de prevención fueron más eficaces cuando las muestras se subdividieron en grupos de alto y bajo riesgo de TCA. De modo que evaluar previamente a los participantes de los programas de 
prevención mediante el protocolo ASEBA prevención puede mejorar su eficacia. Además, se conseguiría una prevención más completa de la psicopatología ya que se trabaja también la prevención de comorbilidades de todos los trastornos que se engloban en la dimensión de INT.

En cuanto a las implicaciones clínicas, el hecho de saber si una persona con TCA es más INT o EXT puede ayudar al profesional a prevenir una posible comorbilidad en la dimensión en que la paciente tenga una puntuación patológica.

\section{Referencias}

Achenbach, T. M., McConaughy, S. H. y Howell, C. T. (1987). Child/adolescent behavioral and emotional problems: implications of cross-informant correlations for situational specificity. Psychological Bulletin, 101(2), 213-232.

Achenbach, T. y Rescorla, L. (2001). Manual for the ASEBA School Age Forms and Profiles. Burlington, VT: Universidad de Vermont.

Adambegan, M., Wagner, G., Nader, I.W., Fernandez-Aranda, F., Treasure, J. y Karwautz, A. (2012). Internalizing and externalizing behaviour problems in childhood contribute to the development of anorexia and bulimia nervosa-a study comparing sister pairs. European Eating Disorders Review, 20(2), 116-120. doi: 10.1002/erv.1152

Anastasiadou, D., Medina-Pradas, C., Sepulveda, A.R. y Treasure, J. (2014). A systematic review of family caregiving in eating disorders. Eating Behaviors, 15(3), 464-477.

American Psychiatric Association (2013). Diagnostic and statistical manual of mental disorders ( $5^{\mathrm{a}} \mathrm{ed}$.) Arlington, VA: Autor.

Berg, C.J., Rapoport, J.L. y Flament, M. (1986). The Leyton Obsessional Inventory-Child Version. Journal of the American Academy of Child Psychiatry, 25(1), 84-91.

Castro, J., Gila, A., Gual, P., Lahortiga, F., Saura, B. y Toro, J. (2004). Perfectionism dimensions in children and adolescents with anorexia nervosa. Journal of Adolescent Health, 35, 392-398. doi: 10.1016/j.jadohealth.2003.11.094

Corral, S., González, M., Pereña, J. y Seisdedos, N. (1998). Adaptación española del Inventario de trastornos de la conducta alimentaria. En D. M. Garner (dir.), EDI-2: Inventario de trastornos de la conducta alimentaria. Manual (pp. 69-80). Madrid: TEA.

Cosgrove, V. E., Rhee, S. H., Gelhorn, H. L., Boeldt, D., Corley, R. C., Ehringer, M. A., Young, S. E. y Hewitt, J. K. (2011). Structure and etiology of co-occurring internalizing and externalizing disorders in adolescents. Journal of Abnormal Child Psychology, 39(1), 109-123. doi: 10.1007/s10802-010-9444-8

Crespo, C., Carona, C., Silva, N., Canavarro, M. C. y Dattilio, F. (2011). Understanding the quality of life for parents and their children who have asthma: family resources and challenges. Contemporary Family Therapy, 33(2), 179-196. doi: 10.1007/s10591-0119155-5

De la Peña, F., Ulloa, R., Higuera, F., Ortiz, S., Arechavaleta, B., Foullux, C., Nogales, I., Márquez, M., Cruz, E., Martínez, P., Hernández, L. y Domínguez, E. (2002, 22-27 de oct.). Interrater reliability of the Spanish version of the K-SADS-PL [Póster]. American Academy of Child \& Adolescent Psychiatry Annual Meeting, San Francisco, CA.

Del Barrio, V. y Carrasco, M. A. (2004). Adaptación del CDI, Inventario de depresión infantil de Maria Kovacs. Madrid: TEA.

Eaton, N. R., Keyes, K. M., Krueger, R. F., Balsis, S., Skodol, A. E., Markon, K. E., Grant, B. F. y Hasin, D. S. (2012). An invariant dimensional liability model of gender differences in mental disorder prevalence: evidence from a national sample. Journal of Abnormal Psychology, 121(1), 282-288. doi: 10.1037/a0024780 
Fairburn, C. G., Cooper, Z., Doll, H. A. y Welch, S. L. (1999). Risk factors for anorexia nervosa. three integrated case-control comparisons. Archives of General Psychiatry, 56, 468-476. doi: 10.1001/archpsyc.56.5.468

Ferreiro, F., Seoane, G. y Senra, C. (2011). A prospective study of risk factors for the development of depression and disordered eating in adolescents. Journal of Clinical Child and Adolescent Psychology, 40, 500-505. doi: 10.1080/15374416.2011.563465

Flett, G. L., Hewitt, P. L., Besser, A., Su, C., Vaillancourt, T., Boucher, D., Munro, Y., Davidson, L. y Gale, O. (2000). The Child-Adolescent Perfectionism Scale. Journal of Psychoeducational Assessment, 34, 634-652. doi: 10.1177/0734282916651381

Forbush, K. T., Hagan, K. E., Kite, B. A., Chapa, D. A., Bohrer, B. K. y Gould, S. R. (2017). Understanding eating disorders within internalizing psychopathology: a novel transdiagnostic, hierarchical-dimensional model. Comprehensive Psychiatry, 79, 40-52. doi: 10.1016/j.comppsych.2017.06.009

Forbush, K. T., South, S. C., Krueger, R. F., lacono, W. G., Clark, L. A., Keel, P. K., Legrand, L. N. y Watson, D. (2010). Locating eating pathology within an empirical diagnostic taxonomy: evidence from a community-based sample. Journal of Abnormal Psychology, 119(2), 282-292. doi:10.1037/a0019189

Forbush, K. T. y Watson, D. (2013). The structure of common and uncommon mental disorders. Psychological Medicine, 43(1), 97-108. doi: 10.1017/S0033291712001092

Garner, D. M. (1991). Eating Disorder Inventory-2 Professional Manual. Odessa, TX: Psychological Assessment Resources.

Hodge, C. P., Meilleur, D., Taddeo, D. y Frappier, J. Y. (2019). The behavioral and affective profile of inpatient adolescent girls with restrictive anorexia nervosa. Eating and Weight Disorders-Studies on Anorexia, Bulimia and Obesity, 24(4), 645-649. doi: 10.1007/s40519-019-00727-3

Hollingshead, A. B. y Redlich, F. C. (1953). Social stratification and psychiatric disorders. American Sociological Review, 18(2), 163-169.

Jacobi, C., Hayward, C., de Zwaan, M., Kraemer, H.C. y Agras, W. S. (2004). Coming to terms with risk factors for eating disorders: application of risk terminology and suggestions for a general taxonomy. Psychological Bulletin, 130(1), 19-65. doi: 10.1037/0033-2909.130.1.19

Kaufman, J., Birmaher, B., Brent, D., Rao, U. y Ryan, N. (1996). Kiddie-Schedule for Affective Disorders and Schizophrenia, Present and Lifetime Version (K-SADS-PL). Pittsburgh, PE: University of Pittsburgh Medical Center.

Kendler, K. S., Prescott, C. A., Myers, J. y Neale, M. C. (2003). The structure of genetic and environmental risk factors for common psychiatric and substance use disorders in men and women. Archives of General Psychiatry, 60(9), 929-937. doi: 10.1001/archpsyc.60.9.929

Kovacs, M. (1992). Children Depression Inventory, CDI. Toronto: Multi-Health Systems.

Lemos, S. (2003). La psicopatología de la infancia y la adolescencia: consideraciones básicas para su estudio. Papeles del Psicólogo, 24(85), 19-28.

Machado, B. C., Gonçalves, S. F., Martins, C., Hoek, H. W. y Machado, P. P. (2014). Risk factors and antecedent life events in the development of anorexia nervosa: a Portuguese case-control study. European Eating Disorders Review, 22(4), 243-251. doi: 10.1002/erv.2286

Martín, J., Padierna, A., Aguirre, U., Quintana, J. M., Hayas, C. y Muñoz, P. (2011). Quality of life among caregivers of patients with eating disorders. Quality of Life Research, 20(9), 1359-1369. doi: 10.1007/s11136-011-9873-z

McDermott, B., Forbes, D., Harris, C., McCormack, J. y Gibbon, P. (2006). Non-eating disorders psychopathology in children and adolescents with eating disorders: 
implications for malnutrition and symptom severity. Journal of Psychosomatic Research, 60(3), 257-261. doi: 10.1016/j.jpsychores.2005.08.004

Meyer, C., Taranis, L., Goodwin, H. y Haycraft, E. (2011). Compulsive exercise and eating disorders. European Eating Disorders Review, 19(3), 174-189. doi: 10.1002/erv.1122

Mischoulon, D., Eddy, K. T., Keshaviah, A., Dinescu, D., Ross, S. L., Kass, A. E., Franko, D. L. y Herzog, D. B. (2011). Depression and eating disorders: treatment and course. Journal of Affective Disorders, 130(3), 470-477. doi: 10.1016/j.jad.2010.10.043

Mitchell, K. S., Wolf, E. J., Reardon, A.F. y Miller, M. W. (2014). Association of eating disorder symptoms with internalizing and externalizing dimensions of psychopathology among men and women. International Journal of Eating Disorders, 47(8), 860-869. doi: $10.1002 /$ eat.22300

Muratori, F., Viglione, V., Maestro, S. y Picchi, L. (2004). Internalizing and externalizing conditions in adolescent anorexia. Psychopathology, 37(2), 92-97. doi: 10.1159/000078089

Palomera, R., Salguero, J. M. y Ruiz-Aranda, D. (2012). La percepción emocional como predictor estable del ajuste psicosocial en la adolescencia. Behavioral Psychology/Psicología Conductual, 20, 43-58.

Phares, V. (1997). Accuracy of informants: do parents think that mother knows best? Journal of Abnormal Child Psychology, 25(2), 165-171. doi: 10.1023/A:1025787613984

Pike, K. M., Hilbert, A., Wilfley, D. E., Fairburn, C. G., Dohm, F. A., Walsh, B. T. y StriegelMoore, R. (2008). Toward an understanding of risk factors for anorexia nervosa: a case-control study. Psychological Medicine, 38(10), 1443-1453. doi: $10.1017 /$ S0033291707002310

Preti, A., de Girolamo, G., Vilagut, G., Alonso, J., de Graaf, R., Bruffaerts, R., Demyttenaere, K., Pinto-Meza, A., Haro, J. M. y Morosini, P. (2009). The epidemiology of eating disorders in six European countries: results of the ESEMeD-WMH project. Journal of Psychiatric Research, 43(14), 1125-1132. doi: 10.1016/j.jpsychires.2009.04.003

Sardinero, E., Pedreira, J. L. y Muñiz, J. (1997). El cuestionario CBCL de Achenbach: adaptación española y aplicaciones clínico-epidemiológicas. Clínica y Salud, 8(3), 447480.

Seisdedos, N. (1990). Adaptación española del cuestionario: State-trait Anxiety Inventory for Children (STAIC). Madrid: TEA.

Sepúlveda, A.R., Moreno, A., Nova, E., Marcos, A., Carrobles, J. A. y Graell, M. (en prensa). Biological, psychological and familial correlates in eating disorders at onset: protocol and preliminary results of a control-case study (ANOBAS) Actas Españolas de Psiquiatría.

Serrano, F., Barrantes-Vidal, N., Domènech, E., Obiols, J. E. y Subirá, S. (1997). Ideación suicida y sintomatología obsesivo-compulsiva en una muestra de adolescentes. Revista de Psicopatología y Psicología Clínica, 2(3), 265-271. doi: 10.5944/rppc.vol.2.num.3.1997.3848

Smink, F. R., Van Hoeken, D. y Hoek, H. W. (2012). Epidemiology of eating disorders: incidence, prevalence and mortality rates. Current Psychiatry Reports, 14(4), 406-414. doi: 10.1007/s11920-012-0282-y

Spielberger, C. D., Edwards, C., Lushene, R. E., Montuori, J. y Platzek, D. (1973). STAIC State-Trait Anxiety Inventory for Children: Preliminary Manual. Palo Alto, CA: Consulting Psychologists Press.

Theodoratou-Bekou, M., Andreopoulou, O., Andriopoulou, P. y Wood, B. (2012). Stressrelated asthma and family therapy: case study. Annals of General Psychiatry, 11(28), 110. doi: 10.1186/1744-859X-11-28 
Toro, J. y Ezpeleta, L. (2014). La psicopatología del desarrollo. En L. Ezpeleta y J. Toro (dirs.), Psicopatología del desarrollo (pp. 33-52). Madrid: Pirámide.

Treasure, J. y Schmidt, U. (2013). The cognitive-interpersonal maintenance model of anorexia nervosa revisited: a summary of the evidence for cognitive, socio-emotional and interpersonal predisposing and perpetuating factors. Journal of Eating Disorders, 1, 13. doi: 10.1186/2050-2974-1-13

Treutler, C. M. y Epkins, C. C. (2003). Are discrepancies among child, mother, and father reports on children's behavior related to parents' psychological symptoms and aspects of parent-child relationships? Journal of Abnormal Child Psychology, 31(1), 13-27. doi: 10.1023/A:1021765114434

Ulloa, R. E., Ortiz, S., Higuera, F., Nogales, I., Fresán, A., Apiquian, R., Cortés, J., Arechavaleta, B., Foulliux, C., Martínez, P., Hernández, L., Domínguez, E. y de la Peña, F. (2006). Interrater reliability of the Spanish version of Schedule for Affective Disorders and Schizophrenia for School Age Children-Present and Lifetime version (KSADS-PL). Actas Españolas de Psiquiatría, 35(1), 36-40.

Yamamoto, N. y Nagano, J. (2015). Parental stress and the onset and course of childhood asthma. BioPsychoSocial Medicine, 9(1), 1. doi: 10.1186/s13030-015-0034-4

RECIBIDO: 17 de abril de 2020

ACEPTADO: 3 de septiembre de 2020 